\title{
ERRATUM to: Study on axial-flow hydrocyclone for in-situ sand removal of natural gas hydrate in the subsea
}

\author{
Haitao Lin, Yuan Huang and Hualin Wang
}

Published online: 24 March 2021

Original article:

E3S Web of Conferences (AEECS 2021)

Volume 245, 2021

$20215^{\text {th }}$ International Conference on Advances in Energy, Environment and Chemical Science (AEECS 2021)

https://doi.org/10.1051/e3sconf/202124501050

This erratum has been made to correct 1 mistake in the authors' list.

Actually, the third author Hualin Wang should be replaced by Yanhong Zhang, with the corresponding author's e-mail "zhyh75@ecust.edu.cn". The correction has been approved by all the authors and the editors.

Study on axial-flow hydrocyclone for in-situ sand removal of natural gas hydrate in the subsea

Haitao Lin $^{1,2}$, Yuan Huang ${ }^{2,3^{*}}$ and Yanhong Zhang ${ }^{1,2^{*}}$

School of Mechanical and Power Engineering, East China University of Science and Technology, Shanghai, 200237, China

${ }^{2}$ National Engineering Laboratory for Industrial Wastewater Treatment, East China University of Science and Technology, 200237, China ${ }^{3}$ School of Environmental and Chemical Engineering, Shanghai University, Shanghai, 200444, China

•Corresponding author's e-mail: yuanhuang@ecust.edu.cn, zhyh75@ecust.edu.cn

\footnotetext{
*Corresponding author's e-mail: yuanhuang@ecust.edu.cn, zhyh75@ecust.edu.cn
} 